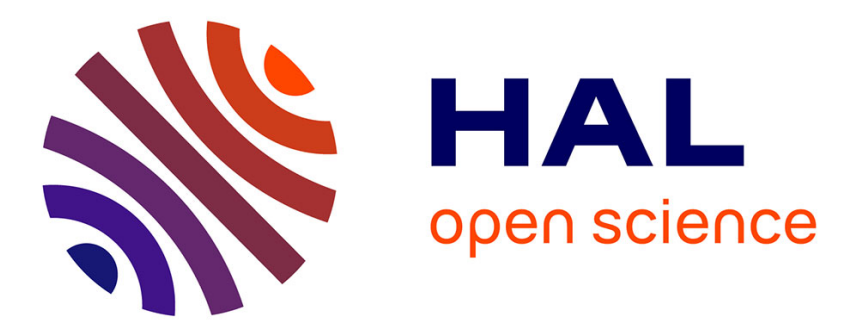

\title{
Technical requirements for the operation of microgrids in both interconnected and islanded mode Working group CIRED 2018-03
}

\author{
Vincent Debusschere, Candelaria Utrilla, Noemi Alonso Palacios, Stefano \\ Mandelli, Lukas Sigrist, Ricardo André
}

\section{To cite this version:}

Vincent Debusschere, Candelaria Utrilla, Noemi Alonso Palacios, Stefano Mandelli, Lukas Sigrist, et al.. Technical requirements for the operation of microgrids in both interconnected and islanded mode Working group CIRED 2018-03. CIRED 2021, Sep 2021, Genève, Switzerland. hal-03369281

\section{HAL Id: hal-03369281 \\ https://hal.science/hal-03369281}

Submitted on 7 Oct 2021

HAL is a multi-disciplinary open access archive for the deposit and dissemination of scientific research documents, whether they are published or not. The documents may come from teaching and research institutions in France or abroad, or from public or private research centers.
L'archive ouverte pluridisciplinaire HAL, est destinée au dépôt et à la diffusion de documents scientifiques de niveau recherche, publiés ou non, émanant des établissements d'enseignement et de recherche français ou étrangers, des laboratoires publics ou privés. 


\title{
Technical requirements for the operation of microgrids in both interconnected and islanded mode Working group CIRED 2018-03 \\ Vincent Debusschere $^{I^{*}}$, Candelaria Utrilla ${ }^{1}$, Noemi Alonso Palacios ${ }^{2}$, Stefano Mandelli ${ }^{3}$, Lukas Sigrist ${ }^{4}$, Ricardo André ${ }^{5}$
}

\author{
${ }^{1}$ Univ. Grenoble Alpes, CNRS, Grenoble INP, G2Elab, 38000 Grenoble, France \\ ${ }^{2}$ i-DE Redes Inteligentes - Iberdrola Group, 48003 Bilbao, Vizcaya, Spain \\ ${ }^{3}$ Consulting, Solutions and Services Division, CESI S.p.A. Milan, Italy \\ ${ }^{4}$ IIT (Institute for Research in Technology), Universidad Pontificia Comillas, 28015 Madrid, Spain \\ ${ }^{5}$ E-Redes, Lisboa, Portugal \\ * vincent.debusschere@grenoble-inp.fr
}

Keywords: WORKING GROUP CIRED, MICROGRIDS, INTERCONNECTED, ISLANDED, OPERATION

\begin{abstract}
Considering the significant increase in projects and initiatives in Europe on microgrids connected to the distribution grid, CIRED launched a working group to address the topic of the technical requirements for the operation of microgrids in both interconnected and islanded modes. The work of this working group started in January 2019 and finished in February 2021. This paper summarizes the content of the report. It aims at providing recommendations on lessons learned from a selected representative and complementary panel of real-life existing demonstration (mostly full scale). The group also took advantage of past projects from where real site conclusions were obtained as well as the expertise within the working group, to guarantee a report with practical conclusions on top of a synthetic state of art. Several microgrid demonstration were identified as top tier projects, covering a wide scope of applications. Among others, the WG studied the deployed assets, the modes of operation, the obtained results and conclusions and mostly the technical lessons learned, i.e., what would be needed to generalize such demonstrator in a daily life operation of a distribution grid (mostly excluding economic, social and environmental criteria).
\end{abstract}

\section{Introduction}

Microgrids may offer significant advantages for end-users and utilities in the current context of energy transition. Territories served by traditional distribution grids up until now could benefit from relying on an intermediary distributed management system at the scale of microgrids. More specifically, microgrids able to operate both connected to the main grid and in islanded mode could improve the resiliency and quality of service of current distribution grids. Interconnection of microgrids could be a sophisticated solution in emergencies and restoration of distribution grids after severe events, but also propose an alternative solution for continuity of supply during maintenance. Lastly, microgrids could be elementary cells that would accompany the energy transition and the evolving distribution grids facing an increase in renewable energy penetration as well as in non-conventional loads, like electric vehicles.

The introduction of these microgrids would however impact the control, operation and protection schemes of distribution systems. Accordingly, technical solutions must be developed, to ensure that the microgrids does not harm people safety, power quality or costs performance.

\section{Objective of the working group}

The main goal of this working group was to build a document gathering the technical requirements needed to properly introduce the concept of microgrids in distribution grids in which the decentralized generation increases significantly and the communication infrastructure is offering new possibilities, as illustrated in Fig. 1. These requirements concern the monitoring and control infrastructure, the protection systems and the design of less traditional components such as inverter-based generators (IBG) that will likely dominate microgrids.
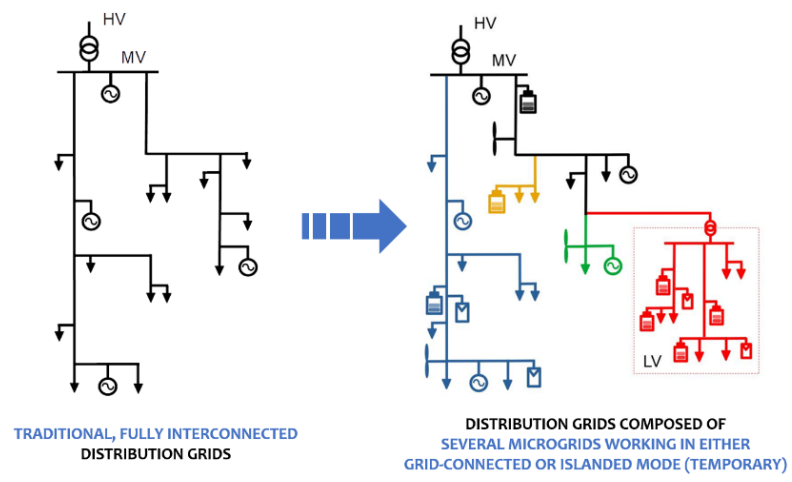

Fig. 1. Distribution grids evolving in temporary microgrids. 
The notion of microgrid was understood as a portion of a distribution grid (thus mostly electrical energy based) owned by a distribution system operators (DSO). The considered microgrid is normally operated in grid-connected mode and could disconnect for various reasons and a range of durations. DC microgrids were not considered in this work as well as multi-energy vector energy systems (even if partly considered in the demonstrators). Subjects needing further investigation were provided in the report when it was not possible to cover them properly (due to time limitation, lack of expertise in the working group and so on).

The idea was to provide, with the report, practical guidelines to DSO and other involved stakeholders, to anticipate and participate to the future microgrids operational standards.

Many questions arise when considering islanding part of a distribution grid. Once defined the area to disconnect, for instance with the help of long-term planning studies, the criteria motivating the transition, the technical capacity to realize it as well as the possible usages of such flexibility are to be discussed. This means being able to define why, when, how and in what conditions transitions from/to grid-connected to/from islanded modes are possible and need to be operated.

Only technical aspects are considered in the report. Technical issues of purely isolated microgrids (e.g., off-grids), like small-signal and transient stability, voltage and frequency control, fault ride through as well as power and energy management systems are out of the scope of this report. The same is true for virtual power plant infrastructures based for instance on self-regulation of dispatchable loads. Indeed, those topics are not specific to grid-connected microgrids transitioning to islanded mode on a temporary basis. For those dedicated topics, the major references from the literature are cited in the report for information. Finally, the economic issues related to the discussion presented in this report were shifted in the scope of a parallel CIRED working group (2019-2), whose report is recommended in complement to the present one.

\section{Content of the report}

The report has been divided in three main parts of equal interest: demonstrators assessment, state of art and recommendations. The final report contains the following chapters:

1. Scope of the work and main definitions: where the context of the work is set and the terminology summarized. The main terms used in the report are defined based on the views of the working group members (for instance grid-forming and grid-following, or isolated and islanded, and so on).

2. Illustration and lessons learned from selected demonstrators: where interviews of what has been tested in a selection of representative and complementary life-scale demonstrators are assessed.

3. Operating microgrids in grid-connected and islanded modes: where are proposed the industrial state of art of criteria, methods and needs to isolate part of a distribution grid. The role of microgrids in enhancing the resiliency of distribution grids is as well discussed.
4. Technical requirements for microgrids to operate in gridconnected and islanded modes: where conclusions are drawn from the previous chapters and recommendations are listed based on the expertise of the working group, per category of technical devices and infrastructures. Further works are also highlighted here.

The following sections present the main elements discussed in the final report, emphasizing the key messages, main conclusions, some recommendations and perspectives of work still to be addressed after the end of the working group.

\section{Lessons learned from demonstrators}

The chapter on demonstrators proposes illustrations and lessons learned from a set of demonstrators. The idea was not to propose a list of all existing demonstrators but rather to limit their number to allow going deeper into their individual assessment. Thus each demonstrator covers different aspects of the scope of the working group and members of the working group have conducted one-to-one interviews with projects manager (when possible targeting technical and operative persons) on that complementary selection of demonstrators. An extensive set of questions was used as a common ground for interviews, which canvas is proposed in the appendix of the report.

The presentation of each demonstrator follows the same structure, ranging from its technical characteristics and design (Automation and control, protection, monitoring, supervision and telecommunication) to the technical actions needed for the operation of the islanding use case, including some relevant experimental results for illustration purposes. The main themes considered in this chapter are listed here:

1. Context and objectives of the demonstrator;

2. Demonstrator grid composition;

3. Equipment used for the islanding use case;
(a) Automation and control;
(b) Protection;
(c) Measurement;
(d) Supervision;
(e) Telecommunication;

4. Modes of operation of the islanding use case;
(a) Grid-connected operation;
(b) Transition from grid-connected to islanded operation;
(c) Islanded operation;
(d) Transition from islanded to grid-connected operation;
(e) Black-start;

5. Islanding tests results.

The set of demonstrators is listed in Table 1. Illustrations of grid architectures from selected demonstrators are proposed in Fig. 2 and Fig. 3.

This chapter concludes with a section on the lessons learned from the demonstrators, specifically focusing on three main aspects: the grid composition, the equipment used for the islanding transition and the available modes of operation. The lessons learned target demonstrators and project managers and do not constitute a requirement list to operate microgrids 
Table 1 Selected demonstrators for detailed assessment.

\begin{tabular}{lcc}
\hline Name & Country & Project \\
\hline San Agustín & Spain & N.A. \\
Caravaca & Spain & $2017-2019$ \\
Grid4EU Vrchlabí & Czech Rep. & $2011-2016$ \\
Grid4EU Nice Grid & France & $2012-2016$ \\
INTERFLEX Nice Smart Valley & France & $2017-2019$ \\
SENSIBLE Évora & Portugal & $2015-2018$ \\
\hline
\end{tabular}

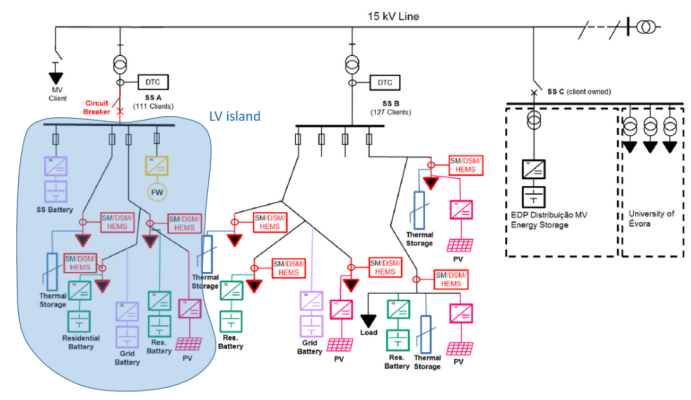

Fig. 2. Évora demonstrator - Grid architecture [2].

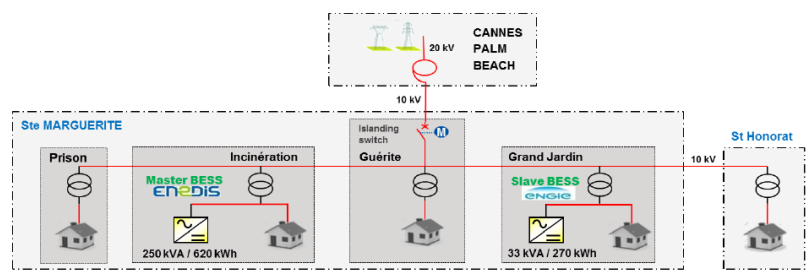

(a)

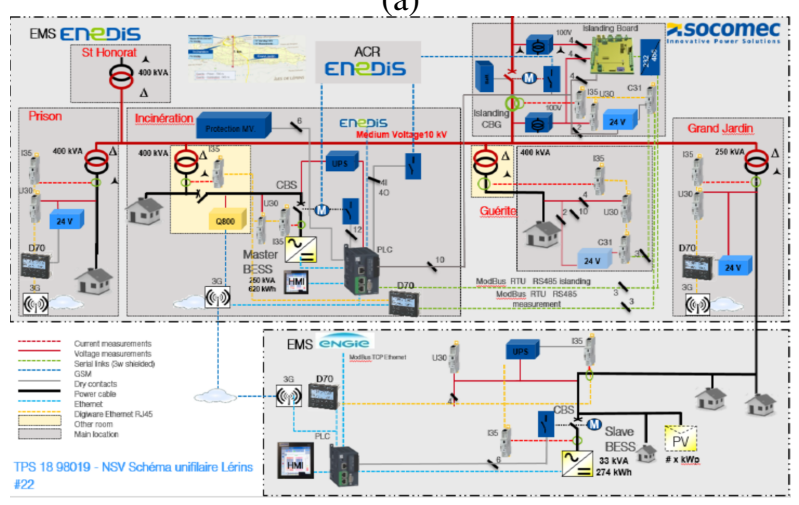

(b)

Fig. 3 Nice Smart Valley demonstrator - Diagram of (a) the distribution grid; (b) the MV grid, including the automation, measurement, telecommunication and supervision [1].

in both grid-connected and islanded modes, though they are meant to complement the recommendations and requirements proposed in the last chapter of the report.

\subsection{Grid composition}

Most of the demonstrators relied on at least one battery facility to build a grid-forming unit while islanded. This opened a series of technical issues, stressing the need for a higher standardization. Although the battery energy systems are theoretically low maintenance, they required constant monitoring, forcing grid operators to focus on management issues rather than on technical/research aspects (to make everything work around the storage facility, like cooling, power electronics, protections, and so on).

Some difficulties arose in that context to find providers offering a complete and finished product, thus affecting the operational teams: they had in fact to learn the competences. Even if outside of the scope of the working group, regulatory issues have been highlighted as well as the absence of economic viability, judging by the current capital costs of storage facilities.

Most of the demonstrators had local photovoltaic production, with some minor disconnection issues. Interestingly, PV inverters already proposed services (not activated by default) that could help stabilizing the microgrid once islanded (a built-in $P / f$ droop notably). Only one studied demonstrator relied a multi-energy unit (combined heat and power) that presented interest though had to be tuned for the experiment. That aspect of islanded microgrids should be further investigated, notably coupled with its economic viability, which seems to be encouraging.

\subsection{Equipment used for the islanding use cases}

The control and automation architectures of the microgrids implemented in the analysed demonstrators were varied, ranging from low to high automation degree (from mostly manual operations to a wide set of automation equipment distributed throughout all the islanded area). An architecture with a low automation degree can be deployed at lower cost, but architectures with a high automation degree allow implementing advanced functionalities, like changing the settings of the grid protections or implementing a hierarchical control of the island voltage and frequency. This would alleviate the tasks of the DSO but at a cost that should be justified by a frequent use of the islanded mode (i.e. for more than just extreme events).

One minor issue has been detected on the supervision of storage facility, because the system was not prepared to represent them (it is neither a producer nor a load).

All the analysed demonstrators were developed in previously existing grids, in which the protection schemes were designed for grid-connected operation. Therefore, these schemes had to be revised to ensure the protection of people and of grid assets in islanded operation. The connection of the neutral to the ground was for instance considered at various voltage level for fault detection. Short-circuit current may be considerably lower in islanded operation than in grid-connected operation, in consequence the protection scheme installed for grid-connected operation may not actuate. The selectivity of the protection scheme could also be perturbed by either the islanded or the grid-connected operation, but maintaining the selectivity was not always essential in the scope of demonstrator, which could not be the case in operational situations. 
Telecommunication solutions differ from one demonstrator to the other. Most of the choices were linked to the particularity of the experimental installations, to ensure the operation of the demonstration with minimal risks. As no analysed demonstrator was dedicated to telecommunication, the working group could not provide strong lessons learned in this report. This should however constitute further investigations.

\subsection{Modes of operation of the islanding use case}

A seamless transition from grid-connected to islanded operation was achieved in all the analysed demonstrators (i.e. power cuts are avoided and all the loads remain connected), with or without ensuring the active and reactive power balanced of the island before the opening of the circuit-breaker. Fig. 4 proposes an illustration from one of the demonstrators assessed in the chapter of such transition of the voltage and current of the grid-forming unit of the microgrid. A seamless transition from islanded operation to grid-connected operation was also experimented in most of the demonstrators, relying typically on standard commercial synchrotacts or sometimes nothing (accepting a rougher transition). Fig. 5 illustrate that from the last demonstrator assessed in this chapter.

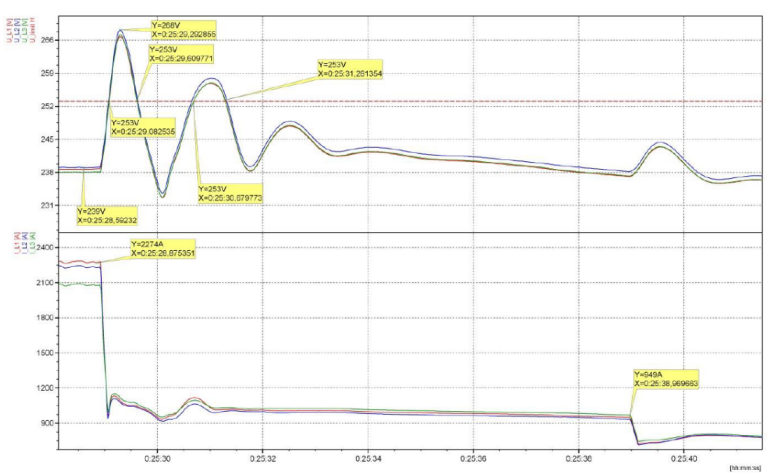

Fig. 4 Vrchlabí demonstrator - Voltage and CHP current curves recorded at the beginning of the islanding test [3]. The measurements were taken in the CHP facility, on the LV side.

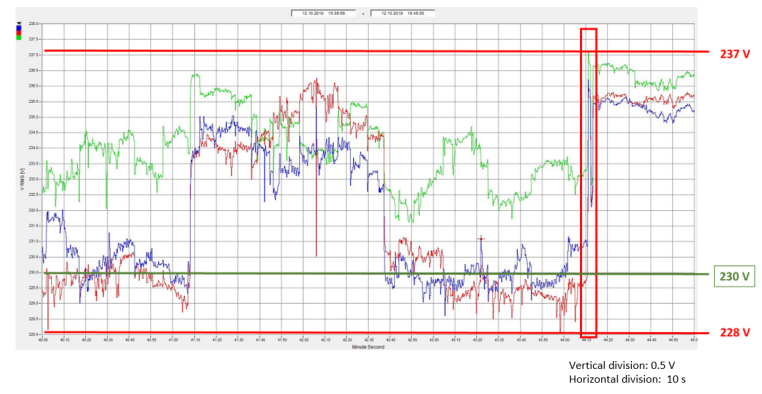

Fig. 5 Évora demonstrator - Voltage curves recorded at the end of the islanding test (synchronized reconnection to the main grid) [4].
Black-start capabilities were successfully implemented in some demonstrators, but also disabled in others, to avoid exposing maintenance operators to electric risk, knowing that they may not yet be prepared to handle elements that can energize the grid autonomously.

Lastly, in almost all the test results shown, the voltage and the frequency are kept within acceptable limits during the transitions and in islanded operation, as illustrated in Fig. 4 and Fig. 6. This did not seem to be a concern in the analysed demonstrators. On the contrary, it was sometimes noted that the voltage and frequency stability was better in islanded than in grid-connected operation.

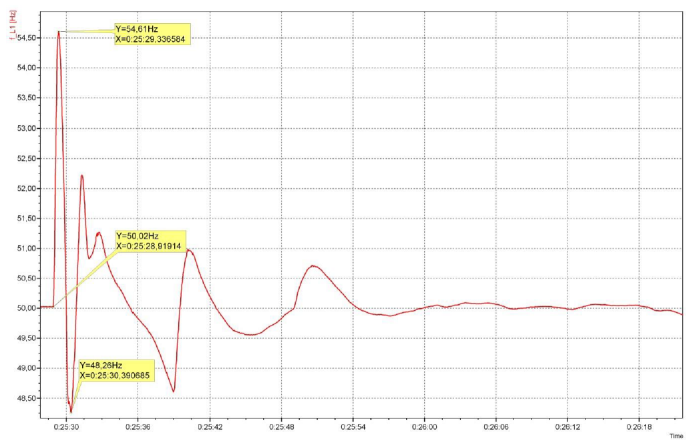

Fig. 6 Vrchlabí demonstrator - Frequency curve recorded at the beginning of the islanding test [3].

\section{Operating microgrids in multiple modes}

The next chapter is mostly focusing what is already out there (standards, industrial application, internationally recognised scientific publications, and so on). It discusses the technical aspects of the two-ways transition between islanded and gridconnected operation of microgrids that are part of a distribution grid. This is done mostly from a state of art perspective, using extensive references to existing standards, grid codes, task forces technical reports and scientific literature. Are presented in this chapter what is already available with no or limited industrial developments.

Resilience and reliability of power systems are defined to illustrate the relevance of considering microgrids that can be operated in islanded mode for a while. The main criteria justifying disconnecting from the main grid are discussed, making the way for flexible microgrids. The chapter also covers technical constraints for microgrids to handle the transition to islanded mode, and then to reconnect to the main grid. Discussions range from control systems to protections, including grid-forming components, and monitoring, control and communication systems.

In most of the presented technical aspects, the conclusion is that existing devices are usually almost sufficient, and could be improved to fulfil the needs: the technical challenges are in range of industrial developments and standard for basic operation of islanded microgrids. The economic viability of such installation does not seem to be guaranteed, even without considering innovative developments, helping increasing 
the reliability of those future temporary microgrids. This means that profitability may not be the main criteria leading to the development of such solutions. Social and environmental criteria could represent significant levers to compensate economic ones, assuming that technical constraints are raised, which is clearly within reach, as proof of concepts were demonstrated already a few years ago.

\section{Technical requirements for microgrids to operate in grid-connected and islanded modes}

The recommendation chapter focuses on the needs to allow part of distribution grids to transition smoothly from grid-connected to islanded modes (and vice-versa), under different (planned and unplanned) operational occasions and not only in lab and life-scale experiments. Indeed, most of the existing demonstrators have been performed at industry sites, campuses, small remote grids, or small islands, all of which to a large degree self-sufficient. The complexity of operating a part of a distribution grid as a microgrid lies in the fact that everything still needs to work as before and, by default, no changes at the customers' sites are allowed.

The main driver of this chapter was to discuss technical solutions that can be of benefit for the operator to handle the changing demands on the distribution grids with the help of microgrids that can switch from grid-connected to islanded modes. The technical recommendation are discussed following the physical layer (protections, power electronics, grid-forming units, power quality) and the information layer (monitoring and control). Most of them do not need to be validated all the time or in all situations. They rather underline general issues.

The standardization of the operational constraints regarding components connected to islanded microgrids still needs updates regarding protections, interoperability, local control connection settings, communication and control for ancillary services, etc. Models of inverters and storage facilities remain a problem for grid operators to study the stability of the future microgrid and its safe operation before and after the islanding. Online characterization tools could be a workaround but the possibility for the manufacturer to provide models to some extend (even in a "black-box" format) would be relevant.

The presented aspects of the chapter do not constitute a mandatory set of requirements to check methodically. Various recommendations are rather outlined to be considered as a function of the operational context. For instance, it should not be necessary to change the whole protection plan if the islanding process is triggered only rarely for maintenance. In other contexts, where the islanding process is activated on a more regular basis, an adaptation of the protection plan would make sense, in addition to clearing safety issues. The time horizon plays a significant role here as well. The islanded operation could last for hours to days, requiring a declination of equipment (from local production to storage facilities).

Market regulations is also something that should play a significant role in how and when this technology can be beneficial to distribution grids operator in the future. However, in most cases it is easier to develop new technology rather than to change regulations, but proper business models are key for social acceptance. To that regard, a parallel CIRED working group is looking into economic viability of microgrids, which report do complement perfectly the current one.

\section{Conclusion}

The main goal of this working group was to build a document gathering the technical requirements needed to properly introduce the concept of microgrids in distribution grids in which the decentralized generation increases significantly and the communication infrastructure is offering new possibilities. These requirements concern the monitoring and control infrastructure, the protection systems and the design of less traditional components such as inverter-based generators that will likely dominate microgrids once islanded. The proposed recommendations target distribution system operators and other involved stakeholders, in order to help them conceive the grid standards and operational rules for the future distribution grids.

A selection of a complementary set of demonstrators in line with the scope of the working group were first analysed in detail. From those technical assessment, lessons learned were drawn. Next, a review of what is already available to distribution system operators was proposed (based on standards, task force reports and recognised scientific literature). Finally, recommendations were gathered in a chapter in the form of what would be technically needed if some transition operation would have to occur in planned scenarios.

The report focuses on microgrids able to operate both in grid-connected and islanded modes. Off-grid systems are not specifically covered although they share common issues regarding the relative high penetration of inverter-based generation that naturally present a low inertia characteristic once isolated, thus challenging their operation. This choice is done knowing that there exist already an extensive literature on the subject, mostly referenced in the report.

Lastly, some elements should be further investigated. In parallel with this working group, another one was working on the economic viability of microgrids, which report is highly recommended. In addition, telecommunication and security could not be properly addressed in this report and would benefit from an in-depth analysis.

\section{References}

[1] Project InterFlex, 'Deliverable D9.3: Demonstration results based on the KPI measurements and lessons learnt from the demonstrations' (ENGIE, Enedis, EDF, GE Grid Solutions, SOCOMEC and GRDF, Dec. 23, 2019).

[2] Project SENSIBLE, 'Deliverable D1.4: Implementation plan for the demonstrators' (EDP Labelec, Sep. 3, 2015).

[3] Project Grid4EU, 'Deliverable D5.4: Demonstration activities results for the Vrchlabí demonstrator (not public)' (C̆EZ Distribuce A.S., Dec. 11, 2015).

[4] Project SENSIBLE, 'Deliverable D4.2: Evora demonstrator (not public)' (EDP Distribuição, Nov. 13, 2018). 